\title{
Sistema de numeración aimara: una revisión para su reconstrucción
}

\author{
Aymara number system: a revision for its reconstruction
}

\author{
Wilfredo Bizarro, ${ }^{1}$ Henry-Mark Vilca-Apaza ${ }^{2}$ y Wilson Sucari ${ }^{3}$ \\ Universidad Nacional del Altiplano de Puno, Perú ${ }^{123}$ \\ ORCID ID: https://orcid.org/0000-0003-0267-6322 ${ }^{1}$ \\ ORCID ID: https://orcid.org/0000-0001-6982-7645² \\ ORCID ID: https://orcid.org/0000-0001-5874-0966 3
}

Recibido: 01 de setiembre de 2020

Aceptado: 15 de noviembre de 2020

\begin{abstract}
Resumen
En este trabajo se revisó la literatura del sistema de numeración aimara, su historia, coexistencias y análisis con respecto a diversas culturas y lenguas, considerando sus tipos, principios y características. En base a ello, se procede a su reconstrucción original del sistema de numeración de la lengua aimara, ya que existen antecedentes y evidencias para afirmar que es un sistema posicional oral, regular, en base decimal, con auxiliar quinaria, además de aditivo y multiplicativo. Se encontró que la raíz primitiva, el número cinco sería qallqu; base originaria al que siguen por adición los demás números: maya '1', paya '2', kimsa '3', pusi '4', qallqu '5', maqallqu (maya qallqu ' $1+5$ '), paqallqu (paya qallqu ' $2+5$ '), kimsaqallqu (kimsa qallqu ' $3+5$ ') y pusiqallqu (pusi qallqu '4 + 5'), y tunka ' 10 ', en función a ella se forman un orden maravillosamente precisa, perfecta desde la interpretación intercultural, lingüística, etnomatemática y matemática.
\end{abstract}

Palabras clave: Matemática, sistema de numeración, aimara, intercultural, etnomatemática.

\begin{abstract}
In this work, the literature of the Aymara numbering system, its history, coexistence and analysis with respect to different cultures and languages was reviewed, considering their types, principles and characteristics. Based on this, it proceeds to its original reconstruction of the Aymara language numbering system, since there is antecedents and evidence to affirm
\end{abstract}


that it is an oral, regular positional system, in decimal base, with quinary auxiliary, in addition to additive and multiplicative. The primitive root number five was found to be qallqu; original base to which the other numbers follow by addition: maya '1', paya '2', kimsa ' 3 ', pusi '4', qallqu '5', maqallqu (maya qallqu '1 + 5'), paqallqu (paya qallqu '2 + 5'), kimsaqallqu (kimsa qallqu ' $3+5$ ') and pusiqallqu (pusi qallqu ' $4+5$ '), and tunka '10', based on it they form a wonderfully precise order, perfect from the intercultural, linguistic, ethnomathematical and mathematical interpretation.

Keywords: Mathematics, numbering system, Aymara, intercultural, ethnomathematics.

\section{Introducción}

La palabra "aimara" deriva de tres palabras compuestas: 1) "jaya", lejano, antiguo, antepasado; 2) "mara", año, años; 3) "aru", voz, lenguaje, hablar. Por lo que sería "jaya mara aru”, o simplemente "aymara", significa "lenguaje de los antepasados" (Deza, 1989; Durand, 1921; Tarifa, 1969). Según Hardman et al. (2001) el aimara pertenece a la familia lingüística jaqi, que incluye a: el jaqaru y el kawki, pues se hablaba en Yauyos que actualmente pertenece en la región Lima provincias, Perú. Existen evidencias arqueológicas y linguiísticas que los idiomas jaqi datan incluso desde la prehistoria. Hubo un tiempo que se hablaba a todo el territorio del Perú actual. Parece que los aimaras "fueron empujados hacia el sur en épocas comparativamente reciente. La separación mayor ocurrió en tiempos del horizonte Huari (400 - 700 d.c.)" (Hardman, et. al., 2001, p. 4). Por su parte Torero (1987) menciona que la lengua aimara forma parte de la familia aru junto con jaqaru y el kauki.

Aimara es la lengua hablada en el altiplano sudamericano abarcando los países de Perú, Bolivia, Chile y Argentina, además en los centros urbanos por movimientos migratorios (González, 1999). Así el antropólogo y jesuita Albó (1995) afirma que este lengua es hablado en Bolivia, Perú, norte de Chile y de Argentina. Se "estima el número de hablantes del aimara en la zona panandina es aproximadamente dos millones" (Mamani, 2007, p. 21). En el tercer concurso somos patrimonio, se afirma que entre 1879 y 1883 Chile conquistó territorios del norte que antes pertenecía a Perú y Bolivia en los que 100\% de la población era Aimara parlantes (Convenio Andrés Bello, 2003). 
La lengua aimara es hasta ahora la segunda más importante del mundo andino y la tercera en importancia numérica entre las lenguas autóctonas de Sudamérica (Bertonio, 1984; Cerron-Palomino, 2000). Llanque (1990) menciona que cuando se inició la expansión incaica en el altiplano, estaba dividido en reinos, entre éstos los más poderosos fueron los Lupacas con su capital Chucuito y los Qollas con su centro Hatuncolla, los otros reinos fueron: Canas, Collaguas, Ubinas, Pacajes, Carangas, Charcas, Canchis, Quillaguas, Omasuyos, Collahuayas, entre otros, que abarcan hoy parte del Cusco, Arequipa y Puno en Perú y La Paz, Oruro, Chuquisaca, Potosí y parte de Cochabamba en Bolivia (Llanque, 1990). Por su parte, De Lucca (1983) en su diccionario Aimara-Castellano refiere que el Aimara es una lengua maravillosa desde el punto de vista lingüístico, bien ordenada y uniforme en la que se encuentra mucho menos irregularidad que en el castellano o cualquier otra lengua europea; cautiva por la lógica y complejidad de su sistema morfológico y sintáctico (De Lucca, 1983). Asimismo, Tarifa (1969) califica que el aimara es idioma perfecto. Además de “clásico, perfecto y admirable” (Vásquez, 2011, p. 36). Por tanto, su sistema de numeración es también perfecta en su formación y maravillosa.

Cerrón-Palomino (2010), investigador de lenguas quechua, aimara, mochica, urochipaya y puquina, manifiesta que todavía subsisten, en relación con el conocimiento de nuestras "lenguas mayores" del antiguo Perú, refiriéndose al puquina, aimara y el quechua.

La historia de las tres lenguas mayores de antiguo Perú: el puquina, el aimara y el quechua, proponiendo los emplazamientos iniciales a partir de los cuales se expandieron hasta confluir en los Andes centro-sureños durante el Período Intermedio Tardío. Proponemos que los incas, a lo largo de su dominación, pasaron por dos etapas de mudanza idiomática: primeramente, del puquina al aimara y, luego, del aimara al quechua. En apoyo de las hipótesis planteadas echamos mano de las evidencias de carácter lingüístico, histórico y arqueológico disponibles (Cerrón-Palomino, 2010, p. 255).

La construcción de los sistemas de numeración en diferentes culturas a través de la historia se desarrolló a partir de las necesidades, vivencias y experiencias. Así muchos pueblos emplean sistemas de numeración decimales. Esto se debe a que tenemos diez dedos en nuestras manos (Perelman, 1973). Por otro lado, había la necesidad de realizar conteo y 
cálculos, ese fue el inicio para crear sus sistemas de numeración y la manera de representar los números (Rojas-Gamarra \& Stepanova, 2015).

\section{Antecedentes históricos antiguos}

Haciendo un análisis de las culturas respecto a sus sistemas de numeración: la cultura egipcia representada por jeroglíficos específicos para cada orden decimal, fue de sistema decimal, se utilizó el principio aditivo y no posicional, escribían de izquierda a derecha o de arriba abajo, modificando la orientación de las figuras según el caso, podían representar números superiores a 106 . El Sistema babilónico fue el primer sistema posicional, (aditivo hasta 60 y posicional para números de orden superior) sexagesimal de base 60, porque a partir del 60 usaron grupos que iban representando el número de unidades, 60, 60x60, 60x60x60 y así sucesivamente. La cultura china utilizó el sistema decimal, representado por ideogramas, incorpora el principio multiplicativo, una mezcla de sumas y productos.

El romano es de tipo no posicional, que incluye el principio sustractivo. Los griegos emplearon dos tipos de numeración, la Ática y la numeración Jónica o alfabética, la Ática fue desarrollada alrededor del año 600 a. C, era de carácter aditivo en base 10, el sistema numeral jónico fue un sistema de tipo aditivo con 27 letras del alfabeto griego.

Las distintas formas de contar de los nuestros pueblos originarios, están íntimamente relacionadas con la estructura lingüística de dichos pueblos (Belloli, 2009). Además el conocimiento de la numeración, varía de una etnia a otra y de una familia linguística a otra (Sánchez, 2009). Así la base del sistema de numeración Yoruba era 20, de los Mayas era 20, y de los Incas, 10. Para Keller (2000) este fue un avance notable en el camino hacia la constitución del sistema de numeración como objeto matemático, puesto que se introduce un principio interno y multiplicativo al desarrollo de la serie numérica (Blanco-Álvarez, 2009). Por otro lado, la lengua chibcha, los chimilas (ette), se rige por un principio de paridad que también establece el lenguaje del parentesco (Le Carrer, 2013).

Respecto a la base cinco, los Khipus serían la escritura numérica de la cultura Inca, la base para escribir el número la Yupana es el 5 y está dado por el conjunto de símbolos (Rojas-Gamarra \& Stepanova, 2015). Las lenguas conocidas como timote-cuicas en la 
literatura lingüística se hablaban en los andes venezolanos, el sistema opera con base decimal, pero también hay evidencia clara de un subsistema quinario (Urban, 2015). El sistema de numeración maya era de base 20 con subbase 5, con uso del cero, fue de sistema aditivo (para los números del 1 al 19) y posicional ( $\mathrm{n} \geq 20$ ), ya que el número uno era representada por un punto, el dos, tres, y cuatro puntos servían para 2, 3 y 4; el 5 representaba una raya horizontal, a ella se añadían los puntos para representar 6, 7, 8 y 9, para diez usaban dos rayas, que continúa hasta el 20 que son cuatro rayas. Según Stanley citado por Fomín (1975), famoso explorador del África, varias tribus africanas empleaban el sistema quinario. Es evidente la relación de este sistema con la forma de la mano del hombre, «máquina computadora» primaria. La lengua aimara como se verá tiene subbase o auxiliar cinco.

\section{Método}

Se realizó una revisión de literatura de los sistemas de numeración de diversas culturas, libros de matemáticas, etnomatemática, artículos científicos, diccionarios de la lengua aimara y demás recursos relacionados con la temática. Se consideró para su análisis los tipos, principios y características de los sistemas de numeración, desde la interpretación intercultural, lingüística, etnomatemática y matemática.

\section{Sistema de numeración}

\section{Definición y base del sistema de numeración}

El sistema de numeración es un conjunto de reglas para expresar y escribir los números (Baer \& Odic, 2019; Baldor, 1997), empleando la menor cantidad de palabras y símbolos (Ministerio de Educación España, 2007), asimismo el sistema de numeración se caracteriza por su base, "que es el número de unidades de un orden que forman la unidad del orden inmediato superior, así en el sistema decimal, la base es 10, porque 10 unidades del primer orden forman una decena, diez decenas forman una centena, etc." (Baldor, 1997, p. 27). Respecto a ello, existen sistemas que se basaron en partes del cuerpo humano como: 5 , 10, 12, 20 y 60. Por ejemplo la base cinco a partir de los dedos de la mano, la base diez utilizando los dedos de las dos manos, la base veinte aparece al utilizar los dedos de las dos 
manos y de los dos pies, la base doce a partir de contar los falanges, la base sesenta aparece como una combinación de cinco y doce. Cabe mencionar que: "los sistemas orales pueden usar más de una base en un solo sistema de numeración” (Pilares, 2005, p. 155).

\section{El sistema actual de numeración decimal}

El sistema de numeración decimal y posicional es el más utilizado en la actualidad. Según Cid et al. (2003) el sistema hindú que se desarrolló al norte de India desde el siglo III a. c. que posteriormente fue asumida por los árabes, quienes han difundido por todo su imperio, por medio de los contactos comerciales y culturales, incluso "entró en competencia con el sistema de numeración romano. Lentamente fue ganando adeptos hasta que a finales del siglo XVIII" quedó definitivamente implantado (Cid et al., 2003, p. 186). Éste es un sistema de numeración escrito: siendo un "sistema posicional regular de base 10. Los símbolos que se definen como sabemos son: 0, 1, 2, 3, 4, 5, 6, 7, 8 y 9" (Cid et al., 2003, p. 188).

Por otro lado, es Sistema de numeración oral con algunas irregularidades como veremos. Asimismo, es un sistema multiplicativo y de base 10 con irregularidades. "Es un sistema multiplicativo porque define símbolos no sólo para los números anteriores a la base sino también para la base y sus potencias" (Cid et al., p.189 ). El número 2400 no lo leemos como "dos cuatro cero cero" sino como "dos mil cuatrocientos", es decir, hacemos referencia a las potencias de la base "mil" y "cien" o "ciento". Dichas irregularidades dependen del idioma y en castellano es: a) once, doce, trece, catorce y quince. En un sistema regular se diría: “dieciuno, diecidos, diecitrés, diecicuatro y diecicinco. b) veinte, treinta, cuarenta, cincuenta, sesenta, setenta, ochenta, noventa. En un sistema regular diríamos: dos dieces (o dos decenas), tres dieses, cuatro dieses, etc. c) Quinientos se diría cinco cientos". (Cid et al., p.189).

\section{Otros sistemas basados en objetos, partes del cuerpo y mixtas}

Cid et al. (2003) presenta además sistemas de numeración basados en la colección de objetos: huesos prehistóricos con muescas, objetos ensartados en hilos (collares), objetos ensartados en varillas (ábacos), caso de los incas representaban números y contaban haciendo nudos en una cuerda teniendo en cuenta la posición en que estaban situados, éstos "indicaban 
unidades, decenas, centenas, millares, etc., a estas cuerdas se les llamaba quipus" (Cid et al., p.192). El mejor ejemplo del método de simbolización es el quipu, un sistema de nudos usado por los incas y bien documentado (Bishop, 1999).

Uno de los estudios más exhaustivos de los sistemas de contar se llevó a cabo en Papúa Nueva Guinea y fue comunicado en primer lugar en Lancy (1978) y también en Lancy (1983). Basándose en los diversos recursos de las dos universidades allí existentes, Lancy pudo alcanzar 225 sistemas de contar que fueron agrupados en los cuatro tipos de sistemas siguientes: Tipo 1: Sistemas basados en contar partes del cuerpo, con el número de partes variando de 12 a 28. Tipo 2: Sistemas que emplean piezas como, por ejemplo, varillas. La base numérica suele estar entre 2 y 5, Tipo III: bases mixtas de 5 y 20 que emplean nombres de números compuestos como "dos manos y un pie" para denotar 15 y Tipo IV: sistemas de base 10 con varios nombres discretos para los números en vez de nombres compuestos" (Bishop, 1999, p. 45).

\section{Sistemas de numeración de las lenguas cercanas al aimara}

En el altiplano sudamericano han existido, coexistido y existen diferentes lenguas como: Puquina, kallawaya, aymara, quechua, uriquilla, ochozuma, cunza, huarpe, uru chi'mu, uru chipaya, uro iruitu y todas las variedades uru, algunos extintos, pero que están en proceso de recuperación, aquí se tiene el sistema de numeración de algunas de estas lenguas:

\section{Uru chipaya}

Cerrón-Palomino (2006) respecto a numeralia dice:

al igual que en el puquina y el quechua, el sistema numeral del chipaya es decimal, sin embargo, de los números cardinales tomados del aimara: (tshii 'uno', pizk 'dos', chhep 'tres', paqpik 'cuatro', phisqa 'cinco', sujta 'seis', paqallaqu 'siete', kimsaqallaqu 'ocho', llatunka 'nueve', tunka 'diez', más abajo en nótese proporciona los números cardinales de iru-wit'u: Chhi 'uno', piski 'dos', chhip 'tres', pajpiku 'cuatro', tojsnuku 'cinco', tajchuru 'seis', tunk 'siete' qunku 'ocho', sanqi 'nueve', qhalu 'diez'” (p. 104).

\section{$\underline{\text { Puquina }}$}

Según Torero (1987) el sistema de numeración puquina es: hukstu 'uno', su 'dos', kappa 'tres', sper 'cuatro', takpa 'cinco', chichu 'seis', stu 'siete', kinas 'ocho', lcheka 'nueve', 
skata 'diez'. Otra fuente: pesq 'uno', so 'dos', qapa 'tres', sper 'cuatro', taqpa 'cinco', chichun 'seis', stu 'siete', kina ‘ocho', cheqa 'nueve', sqaqa ‘diez' (Aguiló, 2000).

\section{$\underline{\text { Kallawaya }}$}

Es una variedad lingüística por herbolarios-curanderos ambulantes de Charazani en actual Bolivia y según Girault (1989) se tiene: uski 'uno', suu 'dos', kapi 'tres', pili 'cuatro', chisma 'cinco', tama 'seis', kajsi 'siete', wasa 'ocho', huki 'nueve', jocha 'diez'. En este caso, Aguiló (2000) hace una comparación de los cinco principales idiomas andinos como se observa en la tabla 1.

\section{Tabla 1}

Comparación interlingüística de los numerales

\begin{tabular}{llllll}
\hline Número & Puquina & Qallawaya & Uru & Quechua & Aimara \\
\hline 1 & Pesq & Uksi & Shi & Uj & Maya \\
2 & So & Soo & Pisqe & Iskay & Paya \\
3 & Qapa & Qapi & Chep & Kimsa & Kimsa \\
4 & Sper & Pili & Pakpik & Tawa & Pusi \\
5 & Taqpa & Chisma & Paanuqo & Pisqa & Pisca \\
6 & Chichun & Tajwa & Pachuy & Sojta & Sojata (qallqo) \\
7 & Stu & Kajsi & Tohonqo & Kanchis & Paqallqo \\
8 & Kina & Wasa & Qonqo & Pusaj & Kimsa qallqo \\
9 & Chepa & Nuki & Sanqaw & Isq'on & Lla-tunqa \\
10 & Sqara & Jocha & Qalo & Chunqa & Tunqa \\
\hline
\end{tabular}

El autor hace una contrastación de los numerales, manifiesta que hay cierto cognatismo lingüístico entre el Puquina y Qallawaya, estos puntos de convergencia aparecen en la nomenclatura de 2 =so-soo y el 3 = Qapa - Qapi (Aguiló, 2000). Respecto al curioso fenómeno de comunicación idiomática del quechua en los números $3=k i m s a$, el $5=$ Pishqa y 6 = sojta, afectando exclusivamente el aimara, finalmente el número 10 en el quechua chunka y el aimara tunca evidencia un cognatismo idiomático (Aguiló, 2000).

\section{Sistema de numeración paralelas al quechua y aimara}

"Los numerales léxicos del quechua y del aimara se pueden clasificar en dos subclases: (a) cardinales y (b) grupales. Seguidamente introduciremos los dos tipos de 
numerales" (Cerrón-Palomino, 2008, p. 191), según se aprecia a continuación en la tablas 2 y 3 .

\section{Tabla 2}

Numerales cardinales en quechua, aimara y español

\begin{tabular}{lll}
\hline Quechua & Aimara & Español \\
\hline Huk & Maya & 'uno' \\
Iskay & Paya & 'dos' \\
Kimsa & Kimsa & 'tres' \\
Tawa & Pusi & 'cuatro' \\
Pichqa & Phisqa & 'cinco' \\
Suqta & Suxta & 'seis' \\
Qanchis & Paqallqu & 'siete' \\
Pusaq & Kimsaqallqu & 'ocho' \\
Isqun & ña (- lla) tunka & 'nueve' \\
\hline
\end{tabular}

\section{Tabla 3}

Numerales de grupo

\begin{tabular}{lll}
\hline Quechua & Aimara & Español \\
\hline chunka & tunka & 'diez' \\
pachak & pataka & 'cien' \\
waranqa & waranqa & 'mil' \\
\hline
\end{tabular}

Como puede apreciarse, señala Cerrón-Palomino (2008, p. 191):

en términos sincrónicos, ambos sistemas numéricos son decimales. No es difícil advertir, sin embargo cuando comparados entre sí, el del aimara muestra dos características, explicables históricamente: (a) un sistema originario de base cinco, es decir quinario, donde se han perdido las raíces primitivas para '3' y '5', pero que para el último se disponía de qallqu, a estar por las evidencias de paqallqu (paya qallqu ' $2+5^{\prime}$ ) y kimsaqallqu (kimsa qallqu ' $3+5$ '); y (b) un reacomodo al sistema decimal sobre la base del molde quechua, lo que supuso, aparte de los préstamos léxicos (kimsa, pichqa y suqta), el recurso a la perífrasis para formar el número nueve, que literalmente significa casi 10.

La interacción milenaria entre las dos familias lingüísticas principales de la región centroandina, la quechua y la aimara, se presenta como uno de los casos de contacto de lenguas más espectaculares que se hayan registrado en el mundo entero. Este contacto destaca 
no solo por sus efectos, ricos en consecuencias, sino también por su larga duración, que podría haber abarcado 2000 años o, inclusive, más tiempo (Adelaar, 2010).

\section{Sistema de numeración aimara}

\section{Sistema de Numeración Aimara utilizado actualmente}

Para poder contar o medir, se requiere del manejo de números y sus cualidades, así los aimaras desarrollaron un sistema numérico muy propio (H. Mamani, 2009).

Arias $(1990,2005)$ presenta analizando los términos en los diccionarios de Bertonio (1984),

De Lucca (1983) y Lucca (1983) y Wilca (1950), tal como se aprecia en la tabla 4:

\section{Tabla 4}

Sistema de numeración aimara según autores

\begin{tabular}{lll}
\hline BERTOÑO (1612) & LUCCA (1983) & PABLO WILCA (1950) \\
Región Lupaca (Altiplano) & Bolivia: La Paz) & Zona: Waraya - Moho \\
\hline Maya (maa) & Maya & Maya (mä) \\
Paya (paa) & Paya (paa) & Paya (pä) \\
Quimsa & Quimsa & Kimsa \\
Pusi & Pusi & Pusi \\
Phisca (pisca) & Phiskca (kallko) & Phisqa (qallqu) \\
Chhukha & Sojjta & Suxta \\
Pacallco & Pakallko & Paqallqu \\
Quimsacallco & Quimsakallko & Kimsaqallqu \\
Llallatunca o ñañatunca & Llatunca & Llatukca o ñatunca-pusiqallqu \\
Tunca & Tunca & Tunka \\
\hline
\end{tabular}

Haciendo una interpretación aritmética, resumiendo las tres fuentes se tendría:

$\begin{array}{ll}\text { Maya (mä) } & \text { Uno } \\ \text { Paya (pä) } & \text { Dos } \\ \text { Kimsa } & \text { Tres } \\ \text { Pusi } & \text { Cuatro } \\ \text { Phisqa o qallqu } & \text { Cinco } \\ \text { Suxta o maqallqu } & \text { Seis } \\ \text { Paqallqu } & \text { Siete } \\ \text { Kimsaqallqu } & \text { Ocho } \\ \text { Llätukca o ñätunca o pusiqallqu } & \text { Nueve } \\ \text { Tunka } & \text { Diez }\end{array}$


Los primeros nueve términos del (del 1 al 9), pueden ser considerados como dígitos; y en su conjunto los diez, como cantidades. Asimismo, en la actualidad en el mejor de los casos no existen referencias de los conceptos originales que nos pueda indicar una relación con objetos o entidades que nos muestren sus raíces. Sin embargo, existen evidencias claras que el número cinco (qallqu) ha sido una base auxiliar en la formación y manejo de los números del sistema aimara. Por otro lado, de acuerdo con las muestras dadas en el cuadro anterior (tabla 4), existe cierta variación en cuanto se refiere a los números cinco, seis y nueve (phisqa, suxta y llätunka). Por lo que es necesario hacer una reconstrucción de números y expresiones actuales que no concuerdan con la deducción etimológica del aimara hablante. Para ello, previamente se establecerá algunas listas de términos del sistema de numeración, dados en el tiempo y espacio del mundo aimara (Arias, 2005, p. 27).

Resulta interesante señalar al paso el uso de un principio sustractivo en la formación de nombre numéricos de los lexemas primarios del aimara por ejemplo $(9=10-1)$. El uso de este principio es inusual, no solo en comparación con el quechua, sino en términos comparativos más globales (Urton \& Llanos, 2003).

\section{“Qallqu” como base auxiliar cinco}

Actualmente, el aimara del sur conserva evidencias de la antigua base quinaria. Los numerales 7 y 8 “son, respectivamente, paqallqu (dos y cinco), kimsaqallqu (tres y cinco). Esta base quinaria coexiste con la base decimal. La prolongada convivencia del quechua y el aimara puede sugerir que una y otra lengua tengan el mismo sistema y los mismos subsistemas componentes" (Pilares, 2005, p. 12). Hurford (1987) citado por Urton \& Llanos (2003) menciona que el aimara muestra evidencias tanto de una base quinaria (base 5, esto es, $2+5=7$ y $3+5=8$ ) como de un principio decimal en la formación de los nombres de números.

Por ello, es necesario averiguar la significación del término "qallqu”, ya que sobre la base de éste se expresan en la lengua aimara los números siete y ocho, es decir, paqallqu y kimsaqallqu respectivamente, y en la actualidad están subyacentes en la expresión viva de los aimara hablantes (Arias, 1990). Según la narración de Wilca ${ }^{1}$ (1950) citado por Arias (2005) "qallqu”, equivale a decir "qallu”, "comienzo de vida o recién nacido". Para ver si esto es posible, hagamos un análisis de las siguientes expresiones en la tabla 5:

${ }^{1}$ Hablante y narrador aimara. 
Tabla 5

Significado del término “qallu” (Arias, 2005)

\begin{tabular}{|c|c|c|}
\hline $\begin{array}{l}\text { Bertoño (1612: 34) } \\
\text { Región Lupaca (Altiplano) }\end{array}$ & $\begin{array}{l}\text { Lucca (1983:217) } \\
\text { Bolivia: La Paz) }\end{array}$ & $\begin{array}{l}\text { Pablo Wilca (1950) } \\
\text { Zona: Waraya - Moho }\end{array}$ \\
\hline $\begin{array}{l}\text { Callu: la mitad, o un lienzo de } \\
\text { cualquier cosa, coserlos: pay } \\
\text { callu cchucuthapitha/cordero y } \\
\text { dizece de todos los animales y } \\
\text { paxaros }\end{array}$ & $\begin{array}{l}\text { Kallu: cría, animal mientras } \\
\text { esté criándose/ una de las dos } \\
\text { piezas que se conforman un } \\
\text { poncho, un awayu, etc. }\end{array}$ & $\begin{array}{l}\text { Qallu: Una de las mitades de } \\
\text { algo que conforma el todo/ } \\
\text { cría de cualquier animal. }\end{array}$ \\
\hline $\begin{array}{l}\text { Anocaracallu; } \\
\text { Cachorro } \\
\text { Callu uta: casa de moxinete } \\
\text { de dos aguas }\end{array}$ & $\begin{array}{l}\text { Kallu uta: Casa de techo con } \\
\text { dos aguas }\end{array}$ & $\begin{array}{l}\text { Qallu uta: casa de techo con } \\
\text { dos aguas iguales }\end{array}$ \\
\hline
\end{tabular}

El término "qallu" en versión de las tres fuentes de información, una de las significaciones que tiene es: "mitad de algo". El número cinco por su parte significa también la mitad o una parte de diez; esto no por pura coincidencia. De manera que el témino "qallu" con el término "qallqu" guarda una aproximación semántica. Asimismo, este último hasta hoy se mantiene formando términos numéricos como "paqallqu" "kimsaqallqu", y no representando el número cinco como debió ser; y que posiblemente, en el decurso tempoespecial es desplazado por el témino "phisqa" (que puede ser témino puquina o quechua). "Por su parte el término "qallu" sigue en vigencia, con una significación muy parecida al témino "qallqu", pero referida a objetos concretos como es la mitad de una frazada o un poncho o un lienzo. Todo esto, en definitiva, nos conduce a sostener que el verdadero término que corresponde en la lengua aimara al número "cinco" es "qallqu", el mismo que en la actualidad ya no se usa como debió usarse anteriormente, por lo que es adecuado y no sólo eso, sino, coherente tomarlo nuevamente y ponerlo en vigencia" (Arias, 2005, p. 28).

Para complementar lo expuesto, Guitel nos dice que el hombre en sus orígenes recurre a las diferentes partes de su cuerpo para contar y que este cuerpo humano ha contribuido a dar una base a la numeración. La mano ofrecía una cualidad muy importante, se le podía considerar como formada por la yuxtaposición de sus cinco dedos, pero no se podía olvidar que la mano formaba un todo. Por su parte, los hombres de la cultura aimara recurrieron a la cabellera, donde a partir de cuatro trenzas pequeñas confluentes a una trenza grande que es la principal, lograron formar y codificar los números: "maya", "paya", 
"kimsa”, "pusi”, “qallqu”, “maqallqu”, "paqallqu”, "kimsaqallqu” y "pusiqallqu". La trenza principal viene a representar al número "qallqu" (cinco), y los de mayor valor a "qallqu" hasta el número "pusiqallqu" inclusive, se forman a partir de éste. Para un mejor entendimiento analicemos la siguiente tabla 6 (Arias, 2005, p. 29).

Tabla 6

Estructura original de numeración aimara

\begin{tabular}{|c|c|c|}
\hline $\begin{array}{c}\text { Bertonio } \\
(1612: 34)\end{array}$ & $\begin{array}{c}\text { Lucca } \\
(1983: 217)\end{array}$ & $\begin{array}{l}\text { Pablo Wilca } \\
\text { (1950) }\end{array}$ \\
\hline *Callu - chatha & Kallu - chatha $i ?$ & Qallu - chatha: \\
\hline Trenzar el cabello. & $\begin{array}{l}\text { Kallu - cha: Trenzas pequeñas de cabello que } \\
\text { luego se unen a dos principales. }\end{array}$ & $\begin{array}{l}\text { Cabellera trenzada } \\
\text { de manera peculiar. }\end{array}$ \\
\hline \multirow{5}{*}{$\begin{array}{l}* * \text { callu - cha: Una } \\
\text { trenza de cabello. }\end{array}$} & Kalluchaña: Parto, etc. / cuando el cabello es un & Kallu - cha: \\
\hline & poco corto, hacerse unas dos o cuatro trenzas & Trenzas pequeñas \\
\hline & pequeñas que luego se unirán a las dos trenzas & de cabello que se \\
\hline & & unen a la trenza \\
\hline & Kallutiyiri: autor, el que es causa de una cosa & principal. \\
\hline
\end{tabular}

El primer caso (*), "qalluchatha" en dos versiones significa la acción de trenzar o entrelazar. $\mathrm{Y}$ en el Segundo caso (**) "qallucha" en las tres versiones existe una aproximación bastante cercana en sus significaciones, pues significa pequeñas trenzas que se unen a la principal, finalmente "Qalluchaña" significa, entre otras, hacerse dos o cuatro trenzas pequeña que luego se unirán a las dos trenzas principales (Arias, 2005). Por otro lado, si consideramos el témino "Kallutiyiri”, cuyo significado es de "autor, el que es causa de una cosa" (Lucca, 1983:217), "qallqu" es originador de los números que le siguen hasta el pusiqallqu". Es decir estas inferencias no son caídas del cielo, sino son el resultado de vivencias experiencias reales y que al codificarlo como número el término "qallqu", en qallque es raíz del verbo "comenzar" o comienzo" se le agrego el sufijo "-qu”, conviertiéndose en nominal y distinguirlo de los demás términos que se asemejan en significación y que corresponde a otros hechos también importantes y que no se refieren al campo matemático (Arias, 2005).

Históricamente el significado del cinco "qallqu" en la cultura aimara tiene mucha importancia, porque el espacio-geopolítico de la nacionalidad aimara estaba 
dividido en cuatro regiones unidas por una capital que viene a ser el centro (taypi) de la organización administrativa de las cuatro regiones asociadas: el pusinti suyo (cuatro regiones del sol) y que en la cultura quechua se denomina como tawantinsuyu. Se debe recordar que la cultura aimara se extendió en el imperio incaico muchos miles de años antes de la cultura quechua y que los primeros incas y toda la región cuzqueña era del habla aimara (Arias, 2005).

En la filosofía aimara pusinti + suyo es representado por una pirámide cuya base es el cuadrado (Arias, 2005). El término "qallqu" que viene a ser la quinta unidad, coincide con la cúspide y centro "taypi", en la que este último viene a ser el equilibrio armónico de las regiones asociadas. Por ello, pusinti significa convergencia de cuatro elementos o concurrencia de políticas, ideas, etc. (Arias, 2005).

\section{Reconstrucción original del sistema de numeración aimara}

Analizando la siguiente tabla 7, vemos que el aimara tiene una regularidad sorprendente en comparación con el castellano.

\section{Tabla 7}

Estructura original de la numeración aimara

\begin{tabular}{llll}
\hline Aimara & $\begin{array}{l}\text { Interpretación } \\
\text { aritmética } \\
\text { sistema aimara }\end{array}$ & Castellano & $\begin{array}{l}\text { Interpretación } \\
\text { aritmética } \\
\text { sistema decimal }\end{array}$ \\
\hline Maya (mä) & 1 & Uno & 1 \\
Paya (pä) & 2 & Dos & 2 \\
Kimsa & 3 & Tres & 3 \\
Pusi & 4 & Cuatro & 4 \\
Qallqu & 5 & Cinco & 5 \\
Maqallqu & $1+5$ & Seis & 6 \\
Paqallqu & $2+5$ & Siete & 7 \\
Kimsaqallqu & $3+5$ & Ocho & 8 \\
Pusiqallqu & $4+5$ & Nueve & 9 \\
Tunka & 10 & Diez & 10 \\
\hline
\end{tabular}

Los números maya (1), paya (2), kimsa (3) y pusi (4), enlazados o antepuestos a qallqu (5), como partes enteras de cinco, de tal manera que forman términos compuestos resultan las expresiones maya qallqu о maqallqu $(1+5)$, paya qallqu о paqallqu $(2+5)$ у 
kimsa qallqu о kimsaqallqu $(3+5)$, Pusi qallqu о Pusiqallqu $(4+5)$, tal como corroboran Arias (2005), Urton (2003), Cerrón-Palomino (2008) y Pilares (2005). En el caso del número tunka (10), como manifiesta Arias (2005) provendría de la lengua uro chipaya "tschuk ${ }^{\mathrm{h}}$ ara" que significa "la segunda mano o las dos manos juntas". Esto enfatiza el uso de las manos en el conteo (Arias, 2005).

\section{Numeración del 11 al 19 en comparación con el castellano}

Una regularidad maravillosamente precisa en cuanto a la oralidad, como se ve en la tabla 8:

\section{Tabla 8}

Estructura original de la numeración aimara del 11 al 19

\begin{tabular}{|c|c|c|c|}
\hline \multirow[b]{2}{*}{ Aimara } & \multirow[b]{2}{*}{\begin{tabular}{l}
\multicolumn{2}{l}{ Interpretación } \\
aritmética sistema \\
aimara
\end{tabular}} & \multirow[b]{2}{*}{ Castellano } & \multirow[b]{2}{*}{\begin{tabular}{l}
\multicolumn{2}{l}{ Interpretación } \\
aritmética sistema \\
decimal
\end{tabular}} \\
\hline & & & \\
\hline Tunka mayani & $10+1$ & Once *Dieciuno & 11 \\
\hline Tunka Payani & $10+2$ & Doce *Diecidos & 12 \\
\hline Tunka kimsani & $10+3$ & Trece $*$ Dieciuno & 13 \\
\hline Tunka pusini & $10+4$ & Catorce *Diecuatro & 14 \\
\hline Tunka qallquni & $10+5$ & Quince *Diecicinco & 15 \\
\hline Tunka maqallquni & $10+6$ & Diecisiés & $10+6$ \\
\hline Tunka paqallquni & $10+7$ & Diecisiete & $10+7$ \\
\hline Tunka kimsaqallquni & $10+8$ & Dieciocho & $10+8$ \\
\hline Tunka pusiqallquni & $10+9$ & Diecinueve & $10+9$ \\
\hline
\end{tabular}

Como se observa en tabla 8, a partir del 11 presenta un sistema de numeración oral regular, con principio aditivo, remitiendonos a Cid et al. (2003) y otros, mientras que en el Castellano presenta irregularidades como, “once, doce, trece, catorce y quince, en un sistema regular se diría: dieciuno, diecidos, diecitrés, diecicuatro y diecicinco" (Cid, et. al. 2003, p. 189). Mamani (2009) manifiesta que esto genera confusiones en el aprendizaje de la numeración, refiriendo a los niños de habla castellana, dicen: "diez y uno", "diez y dos", etc. dentro del aimara, no hay posibilidad de confusión puesto que existe una sola regla para la composición de los números. En esta cualidad de la matemática aimara sugiere la conveniencia de enseñar la numeración a partir de su idioma materno. 
De otra manera, como dice Schroeder (2005), se podría obstaculizar el desarrollo del pensamiento matemático, puesto que los sistemas numéricos de su lengua y del castellano pueden estar basados en lógicas distintas. Según investigaciones se puede comprobar que los niños aimaras y ashánincas obtienen mejores resultados en matemáticas cuando la materia es impartida en el idioma materno y el material de trabajo es adaptado al contexto lingüístico y socio cultural (Mamani, 2009); ellos adquieren un profundo concepto numérico (Schroeder, 2005). Por otro lado, para contar de 10 a más en aimara se le añade el sufijo -ni, tenemos por ejemplo $12=$ tunka payani $($ tunka $=10+$ paya $-n i=2)$.

Numeración del 20 al 29 en comparación con el castellano

\section{Tabla 9}

Estructura original de la numeración aimara del 11 al 19

\begin{tabular}{|c|c|c|c|}
\hline Aymara & $\begin{array}{l}\text { Interpretación } \\
\text { aritmética } \\
\text { sistema aimara }\end{array}$ & Castellano & $\begin{array}{l}\text { Interpretación } \\
\text { aritmética sistema } \\
\text { decimal }\end{array}$ \\
\hline Pä tunka & $2(10)$ & Veinte & 20 \\
\hline Pä tunka mayani & $2(10)+1$ & Veintiuno & $20+1$ \\
\hline Pä tunka Payani & $2(10)+2$ & Veintidos & $20+2$ \\
\hline Pä tunka kimsani & $2(10)+3$ & Veintitres & $20+3$ \\
\hline Pä tunka pusini & $2(10)+4$ & Veinticuatro & $20+4$ \\
\hline Pä tunka qallquni & $2(10)+5$ & Veinticinco & $20+5$ \\
\hline Pä tunka maqallquni & $2(10)+6$ & Veintiseis & $20+6$ \\
\hline Pä tunka paqallquni & $2(10)+7$ & Veintisiete & $20+7$ \\
\hline Pä tunka kimsaqallquni & $2(10)+8$ & Veintiocho & $20+8$ \\
\hline Pä tunka pusiqallquni & $2(10)+9$ & Veintinueve & $20+9$ \\
\hline
\end{tabular}

Numeración de decenas, centenas, unidades de millar, etc. en comparación con el castellano 
Tabla 10

Estructura original de la numeración aimara mayores

\begin{tabular}{|c|c|c|c|}
\hline Aimara & $\begin{array}{l}\text { Interpretación } \\
\text { aritmética } \\
\text { sistema aimara }\end{array}$ & Castellano & $\begin{array}{l}\text { Interpretación } \\
\text { aritmética sistema } \\
\text { decimal }\end{array}$ \\
\hline Tunka & 10 & Diez & 10 \\
\hline Pä tunka & $2(10)$ & Veinte & 20 \\
\hline Kimsa tunka & $3(10)$ & Treinta & 30 \\
\hline Pusi tunka & $4(10)$ & Cuarenta & 40 \\
\hline Qallqu tunka & $5(10)$ & Cincuenta & 50 \\
\hline Maqallqu tunka & $6(10)$ & Sesenta & 60 \\
\hline Paqallqu tunka & $7(10)$ & Setenta & 70 \\
\hline Kimsaqallqu tunka & $8(10)$ & Ochenta & 80 \\
\hline Pusiqallqu tunka & $9(10)$ & Noventa & 90 \\
\hline Pataka & 100 & Cien & 100 \\
\hline Pä pataka & $2(100)$ & Doscientos & 200 \\
\hline Kimsa pataka & $3(100)$ & Trescientos & 300 \\
\hline Mä warang'a & 1000 & Mil & 1000 \\
\hline Pä waranq'a & $2(1000)$ & Dos mil & $2(1000)$ \\
\hline Tunka waranq'a & $10(1000)$ & Diez mil & $10(1000)$ \\
\hline Tunka mayani waranq’a & $11(1000)$ & Once mil & $11(1000)$ \\
\hline Pataka waramq'a & $100(1000)$ & Cien mil & $100(1000)$ \\
\hline Pataka tunkani waranq'a & $110(1000)$ & Ciento diez mil & $110(1000)$ \\
\hline Junu & 1000000 & Millón & 1000000 \\
\hline Pä junu & $2(1000000)$ & Dos millones & $2(1000000)$ \\
\hline
\end{tabular}

En castellano: "veinte, treinta, cuarenta, cincuenta, sesenta, setenta, ochenta, noventa. En un sistema regular se diría: dos dieces (o dos decenas), tres dieses, cuatro dieses, etc" (Cid, et. al. 2003, p. 189). Los demás si presenta regularidad, en cuanto al aimara Mamani $(2009,2013)$ presenta la siguiente tabla 11 sobre la numeración decimal aimara. 
Tabla 11

Numeración decimal aimara

\begin{tabular}{lll}
\hline Número & Aimara & Castellano \\
\hline 0 & Chu'sa & Cero \\
1 & Maya & Uno \\
10 & Tunka & Diez \\
100 & Pataka & Cien \\
1000 & Waranqha & Mil \\
10000 & Tunka waranqha & Diez mil \\
100000 & Pataka waranqha & Cien mil \\
1000000 & Junu & Millón \\
1000000000000 & Junjunu & Billón \\
1000000000000000000 & Junkisawayta & Trillón \\
$10^{24}$ & Junpusiwayta & Cuatrillón \\
$10^{60}$ & Juntunkawayta & Decillón \\
\hline
\end{tabular}

\section{Principio aditivo y multiplicativo del aimara}

El aimara presenta también el principio aditivo y multiplicativo, como se observa en la tabla siguiente (12).

Tabla 12

Ejemplo de principio aditivo y multiplicativo

\begin{tabular}{ll}
\hline \multicolumn{1}{c}{ Según el principio aditivo } & \multicolumn{1}{c}{ Según la segunda regla de multiplicación } \\
\hline Tunka mayani: $10+1=11$ & Paqallqu pataka: $7 \times 100=700$ \\
Pataka kimsaqalquni: $100+8=108$ & Pusiqallqu waranq'a: $9 \times 1000=9000$ \\
Pataka tunkani: $100+10=110$ & Tunka waranq'a: $10 \times 1000=10000$ \\
Pataka tunka payani: $100+10+2=112$ & kimsa tunka waranqa: $(3 \times 10) \times 1000=30000$ \\
Waranq'a pusini: $1000+4=1004$ & Pusi tunka waranqá: $40(1000)$ \\
Waranq'a pataka tunkani: $1000+100+10=1110$ & Waranq'a waranq'a: $1000 \times 1000$ \\
\hline
\end{tabular}

\section{Conclusiones}

El sistema de numeración aimara tuvo un sistema originario posicional oral, regular, en base decimal, con auxiliar quinaria, el qallqu '5', que es la base auxiliar y que por adición siguen los demás números tal como se ha observado: maqallqu (maya qallqu '1 + 5'), paqallqu (paya qallqu ' $2+5$ '), kimsaqallqu (kimsa qallqu ' $3+5$ ') у pusiqallqu pusi qallqu '4 
+5'). Para contar los números a partir de número diez a más se le añade el sufijo -ni, tenemos por ejemplo $12=$ tunka payani (tunka $=10+$ paya $-n i=2)$.

Por ende, la lengua aimara presenta un sistema de numeración oral regular, con principio aditivo a su vez multiplicativo, tal como se muestra en las tablas 8,910 y 11 . Haciendo un análisis comparativo con las otras lenguas, es perfecta en su formación a nivel lingüístico, etnomatemático y matemático; bien ordenada y uniforme en la que no se encuentran irregularidades, tal como anteceden los investigadores. Por tanto, los aimaras desarrollaron un sistema numérico suigéneris. Para el proceso de enseñanza - aprendizaje en la lengua aimara, no hay posibilidad de confusión puesto que existe una sola regla para la composición de los números en comparación con el castellano que obstaculiza el desarrollo del pensamiento matemático, esto según las experiencias de trabajo con niños aimara hablantes.

\section{Referencias}

Adelaar, W. (2010). Trayectoria histórica de la familia linguiística quechua y sus relaciones con la familia lingüística aimara. Boletín de Arqueología PUCP, 14, 239-254. Recuperado de: http://revistas.pucp.edu.pe/index.php/boletindearqueologia/article/view/1334

Aguiló, F. (2000). El idioma del pueblo puquina: un enigma que va aclarándose (Número 1). Intercultural de las Nacionalidades Pueblos Indígenas.

Albó, X. (1995). Bolivia plurilingüe: guía para planificadores y educadores (Vol. 2). UNICEF.

Arias, P. (1990). Niños Aimara aprenden matemática. Universidad Nacional del Altiplano.

Arias, P. (2005). No Etnomatemática en educación primaria. Editorial Titicaca FCEDUC.

Baer, C., \& Odic, D. (2019). Certainty in numerical judgments develops independently of the approximate number system. Cognitive Development, 52, 1-14. https://doi.org/10.1016/j.cogdev.2019.100817

Baldor, A. (1997). Aritmética. Publicaciones Cultural, México.

Belloli, L. A. (2009). Algunos aportes al conocimiento de la numeración Mapuche. Revista 
Electrónica de Investigación en Educación en Ciencias 24(2), 1-7. Recuperado de: http://ppct.caicyt.gov.ar/index.php/reiec/article/view/7383

Bertonio, L. (1984). 1612. Vocabulario de la lengua aymara. Juli, Peru: Francisco del Canto.

Bishop, A. J. (1999). Enculturación matemática: la educación matemática desde una perspectiva cultural (Vol. 49). Grupo Planeta (GBS).

Blanco-Álvarez, H. (2009). Del número a los sistemas de numeración.

Cerron-Palomino, R. (2000). El origen centroandino del aimara. Boletín de Arqueología $\begin{array}{lrl}\text { PUCP, } & \text { 4(4), 131-142. }\end{array}$ http://revistas.pucp.edu.pe/index.php/boletindearqueologia/article/view/2201

Cerrón-Palomino, R. (2006). El chipaya o la lengua de los hombres del agua. Fondo Editorial PUCP.

Cerrón-Palomino, R. (2008). Quechumara: Estructuras paralelas del quechua y del aimara. Plural editores.

Cerrón-Palomino, R. (2010). Contactos y desplazamientos lingüísticos en los Andes centrosureños: el puquina, el aimara y el quechua. Boletín de Arqueología PUCP, 14, 255282.Recuperado de: http://revistas.pucp.edu.pe/index.php/boletindearqueologia/article/view/1335

Cid, E., Díaz-Godino, J., \& Batanero-Bernabeu, M. del C. (2003). Sistemas numéricos y su didáctica para maestros. Universidad de Granada, Departamento de Didáctica de la Matemática.

Convenio Andrés Bello. (2003). Experiencias de apropiación social del patrimonio cultural y natural. emana esperanza para el joven aymara. Extensión metodológica al niño andino Huara.

De Lucca, M. (1983). Diccionario Aymara-Castellano. Comisión de Alfabetización y Literatura en Aymara.

Deza, J. F. (1989). Diccionario aymara-castellano castellano-aymara. Consejo Nacional de Ciencas y Tecnologica.

Durand, J. (1921). Etimologias Perú-bolivianas. Talleres gráficos La Prensa de José L. Calderón.

Fomín, S. V. (1975). Sistema de numeración. Editorial MIR. 
Girault, L. (1989). Kallawaya: el idioma secreto de los incas. Unicef.

González, S. (1999). Interculturalidad y globalización: el caso de los aymaras de Tarapacá. Revista de Ciencias Sociales $(\mathrm{Cl})$, 9, 114-125. Recuperado de: https://www.redalyc.org/pdf/708/70800908.pdf

Hardman, M., Vásquez, J., \& Yapita, J. de D. (2001). Aymara. Compendio de estructura fonológica y gramatical. Universidad Mayor de San Simón.

Keller, O. (2000). Préhistoire de l'arithmétique, La découverte du nombre et du calcul. Ellipse.

Le Carrer, C. (2013). Contar y formar el mundo. Sistema de numeración de los ngäbes de Costa Rica y Panamá. Cuadernos Inter. ca mbio sobre Centroamérica y el Caribe, 10(12), 79-103. Recuperado de: https://revistas.ucr.ac.cr/index.php/intercambio/article/view/12343

Llanque, D. (1990). La cultura aymara: desestructuración o afirmación de identidad. IDEA, Instituto de Estudios Aymaras.

Mamani, H. (2009). Etnomatemática aymara: términos, técnicas y conceptos matemáticos. Asamblea Nacional de Rectores.

Mamani, H. (2013). Etnomatemática aimara: téminos, técnicas y conceptos matemáticos. Aporte a una educación y sociedad intercultural. Corparación MERU E.I.R.L.

Mamani, R. (2007). ¿Primero el castellano y después el aimara? Enseñanza del aimara como segunda lengua en Opoqueri (carangas, Oruro). Tesis de maestría. Universidad Mayor de San Simón. Recuperado de: http://bvirtual.proeibandes.org/bvirtual/docs/tesis/proeib/Tesis\%20Roman\%20Mam ani.pdf

Perelman, Y. (1973). Arimética Recreativa. Ediciones MIR.

Pilares, G. (2005). Los sistemas numéricos del quechua y el aimara. Revista andina, 40, 149178. Recuperado de: http://www.revistaandinacbc.com/wpcontent/uploads/2016/ra40/ra-40-2005-05.pdf

Rojas-Gamarra, M., \& Stepanova, M. (2015). Sistema de numeración Inka en la Yupana y el Khipu. Revista Latinoamericana de Etnomatemática: Perspectivas Socioculturales de la Educación Matemática, 8(3), 46-68. Recuperado de: 
https://www.redalyc.org/pdf/2740/274041587004.pdf

Sánchez, D. (2009). El Sistema de Numeración y algunas de sus aplicaciones entre los aborígenes de Venezuela. Revista Latinoamericana de Etnomatemática, 2(1), 43-68.

Recuperado

de:

https://revista.etnomatematica.org/index.php/RevLatEm/article/view/15

Schroeder, J. (2005). Más allá de los platos típicos: el proyecto matemática intercultural en el Perú. Cuadernos interculturales, 3(4), 51-63. Recuperado de: https://www.redalyc.org/pdf/552/55200405.pdf

Tarifa, E. (1969). Suma lajjra aymara parlaña: Gramática de la lengua aymara. Editorial Don Bosco.

Torero, A. (1987). Lenguas y pueblos altiplánicos en torno al siglo XVI. Revista andina, 5(2), 329-405.

Urban, M. (2015). Notas sobre el sistema de numeración de las lenguas Timote-Cuicas. Boletín Antropológico, 33(90), 53-69. Recuperado de: https://www.redalyc.org/pdf/712/71243364004.pdf

Urton, G., \& Llanos, P. (2003). La vida social de los números: una ontología de los números y la filosofía de la aritmética quechuas (Vol. 2). Centro de Estudios Regionales Andinos Bartolomé de las Casas.

Vásquez, I. (2011). La biblioteca del idioma aymara. Revista de la Biblioteca y Archivo Histórico de la Asamblea Legislativa Plurinacional, 5(15), 33-40. Recuperado de: http://www.revistasbolivianas.org.bo/scielo.php?script=sci_arttext\&pid=S1997$44852011000400005 \& \operatorname{lng}=$ es\&nrm=iso 